\title{
Optimizing an objective function under a bivariate probability model
}

\author{
Xavier Brusset a,1,2,* \\ ${ }^{\mathrm{a}} I A G$, Université Catholique de Louvain, Place des Doyens 1, B-1348 \\ Louvain-la-Neuve, Belgium \\ Nico M. Temme ${ }^{b}$ \\ ${ }^{\mathrm{b}}$ Centrum voor Wiskunde en Informatica (CWI), Kruislaan 413, $1098 \mathrm{SJ}$ \\ Amsterdam, The Netherlands
}

\begin{abstract}
The motivation of this paper is to obtain an analytical closed form of a quadratic objective function arising from a stochastic decision process with bivariate exponential probability distribution functions that may be dependent. This method is applicable when results need to be offered in an analytical closed form without double integrals. However, the study only applies to cases where the correlation coefficient between the two variables is positive or null. A stochastic, stationary objective function, involving a single decision variable in a quadratic form is studied. We use a primitive of a bivariate exponential distribution as first expressed by Downton (1970) and revisited in Iliopoulos (2003). With this primitive, optimization of objective functions in Operations Research, supply chain management or any other setting involving two random variables, or calculations which involve evaluating conditional expectations of two joint random variables are direct. We believe the results can be extended to other cases where exponential bivariates are encountered in economic objective function evaluations. Computation algorithms are offered which substantially reduce computation time when solving numerical examples.
\end{abstract}

Key words: decision analysis, Downton's bivariate exponential distribution, asymptotics of special functions, double integrals, optimization. 


\section{Introduction}

This paper arose from the necessity to solve a specific case in supply chain management. Let us imagine a supplier and a manufacturer engaged in commercial transactions involving the exchange of a vital input for the manufacturer, the price of which is stochastic and stationary. Both supplier and manufacturer are price takers, hence the price fluctuations are exogenous to both actors. This same manufacturer, on the downstream side, must also respond to demand from end customers. This demand fluctuates exogenously in a stochastic stationary fashion. We are interested in problems where demand and price are two variables which impact the manufacturer's cost structure in a supply chain problem. We tend to consider that these situations are amply represented in practice.

Generally, in the literature because the mathematics quickly become intractable, most models cannot represent real world conditions and very often involve just one random variable. We list here some examples of this literature.

Lariviere (1999) models a one period setting in which a monopolistic manufacturer sells to a retailer facing a newsvendor problem. The demand faced by the retailer is an exogenous stochastic random variable. It is shown that, because the manufacturer is a monopolist and because of the elasticity function of standard economics, either price or demand can be chosen as the random variable and the second variable follows from inverting the cumulated distribution function of the first. The author on page 238 of Lariviere (1999) points out that the outcomes would be markedly different in the case of a competitive setting. Using the results presented in our paper, these outcomes could be evaluated using a bivariate exponential distribution for price and demand in a competitive setting.

Wu et al. (2002) model contracting arrangements between one seller and one or more buyers. The seller and buyers can negotiate bilateral contracts in advance and use the spot price reference on the day to complement the contract. An underlying assumption is that the seller is a monopolist, or behaves in a monopolistic way and hence that the final demand is a result of the price practised on the Spot market. Their model would have been greatly enhanced had the authors been able to model two random variables: price on the spot market upstream of the buyer and demand downstream from the buyer. Ex-

\footnotetext{
* Rue Papenkasteel 172, 1180 Uccle, Belgium

Email addresses: xavier@brusset.com (Xavier Brusset ), nicot@cwi.nl (Nico M. Temme).

1 We wish to thank Prof. Philippe Chevalier from IAG and two anonymous referees for their thorough and careful reviews as well as their inspiring comments.

2 This May 2005 version is a complete overhaul from the December 2004 version.
} 
tensions Wu et al. (2001a,b) of Wu et al. (2002) to cases of multi-seller and multi-buyer settings still use the traditional Willingness-To-Pay demand function which denotes that the final demand is a function of the price practised in the spot market for the good being resold.

In Kleindorfer \& Wu (2003), a capacity-constrained seller and a buyer transact through traded options on a B2B (Business-to-Business) and complement these transactions by other transactions through an open Spot market exchange. The good which is being traded is further sold on by the buyer who receives some demand $D_{s}$ which is a function of the spot price observed in the Spot market $P_{s}$. Even though it does not clearly state it, this model basically assumes that the seller is a monopolist and the buyer's demand is competing for constrained capacity from this seller with other buyers in the market. The ultimate demand is only a resultant of the price variable. In the numerical example, the distribution of the spot price follows an exponential distribution. The authors would probably be able to enlarge their results to competitive market settings using the results established in our paper.

In Spinler et al. (2003), the model features a two-period market model with state contingent spot prices and options exchanged by a buyer who needs to satisfy demand and a seller who needs to use available but constrained capacity by selling either on the spot market or through options for future delivery. Once again, because of mathematical complexity, Demand has been modeled to take only two states: either demand can be totally satisfied by exercising the options or it can only be partially satisfied when it exceeds the quantity contracted through options and the buyer of this capacity needs to turn to the spot market for the remaining part.

To our knowledge, supply chain literature involving two random variables is scarce. Seifert et al. (2004) study optimal procurement strategies in a model of spot markets and alternative long term contracts for supplies. In their model, both spot prices and demand follow a stochastic process with known mean and standard deviation. The model further derives the optimal closed-form contract quantity when demand and spot follow a bivariate normal distribution with correlation $\rho \geq 0$.

The other example is Brusset \& Temme (2005) which deals with a special case of a shipper and a carrier who put a contract in place to carry the demand addressed to the shipper. In Brusset \& Temme (2005), the shipper can also turn to the Spot market for transport capacity from alternative carriers at a different price from the contract price. This paper discusses various incentives to coordinate the shipper and carrier's interests and to bridge information asymmetry in an environment where both demand and spot price fluctuate. The present paper's results have been used (including the routines described in appendix B on page 21) to give a numerical example in Brusset \& Temme 
(2005).

In a more general sense, suppose the manufacturer (buyer), as a participant in a competitive market where she is a price taker, must meet some exogenous stochastic stationary demand for her product. Suppose moreover that the buyer must buy some necessary input in an upstream market to be able to meet this demand. Suppose finally that the market for this input is also competitive. Both demand and price are random variables which may or may not be dependent. The manufacturer must deal with uncertainty in both her costs (price of input) and her sales (quantity to be sold). This paper addresses the case where both price and demand follow a bivariate exponential distribution and the objective function involves using both with two decision variables. The correlation coefficient between both random variables must be positive or null. As suggested in Iliopoulos (2003), one of the most important bivariate distributions in both reliability and economic theory is the bivariate exponential of which there are various examples in the literature. We justify choosing this particular bivariate distribution following in the steps of Marshal \& Olkin (1967), namely it would be best for applicability purposes if exponential marginals existed. We have not considered using Gumbel's bivariate because correlation cannot exceed $\pm 1 / 4$ and we think that we may encounter cases of higher correlations in reality. Finally the choice of this bivariate allows for the random variables to be different in nature (which rules out Freund's bivariate). A recent review can be found in Kotz et al. (2000) but we admit not having combed through it extensively. Downton's bivariate exponential distribution (Downton, 1970) has a probability density function (pdf)

$$
f\left(x, y, \lambda_{1}, \lambda_{2}, \rho\right)=\frac{\lambda_{1} \lambda_{2}}{1-\rho} e^{-\frac{\lambda_{1} x+\lambda_{2} y}{1-\rho}} I_{0}\left(\frac{2 \sqrt{\rho \lambda_{1} \lambda_{2} x y}}{1-\rho}\right)
$$

where $x, y, \lambda_{1}, \lambda_{2}$ are positive, $0 \leq \rho<1$, and

$$
I_{0}(z)=\sum_{k=0}^{\infty} \frac{(z / 2)^{2 k}}{k ! k !}
$$

is the modified Bessel function of the first kind of order zero. The above density was initially derived in a different form by Moran (1967). The form (1.1) is derived by Downton (1970) in a reliability context and is a special case of Kibble's bivariate gamma distribution (Kibble, 1941).

Let $(X, Y)$ be an observation from (1.1). The marginal distributions of both $X$ and $Y$ are exponential with means $1 / \lambda_{1}$ and $1 / \lambda_{2}$, respectively. As earlier noted in Iliopoulos $(2003)$, since $I_{0}(0)=1$, it is clear that $X$ and $Y$ are independent if and only if $\rho=0$. Downton (1970) showed that $\rho$ is the correlation coefficient of the two variates. 


\section{Optimization of an objective function}

Whenever a decision maker has to make a management decision and that the outcome of his choice is affected by the realization of a random variable, the equation that represents this interaction between both decision and state of nature is best captured by a partial moment with the decision variable cropping up both inside the integral and at the definition of the boundary. If $C($.$) is the objective function which has to be optimized, f($.$) is the probability$ density function of the random variable $Q$ and $x$ is the variable of decision, then he may have to find $x$ such that

$$
C(x \mid q)=\frac{C(x)}{\operatorname{pr}(Q<x)}
$$

This way of expressing the objective function leads to the expected objective function becoming

$$
E(C(x \mid q))=\int_{0}^{x} C(x) f(q) d q .
$$

In this paper we focus on the interaction between two decision variables and two random variables which leads naturally to this kind of expression:

$$
E(C(x, y \mid q, p))=\int_{0}^{y} \int_{0}^{x} C(x, y) f(q, p) d q d p .
$$

When both random variables are independent, solutions flow straightforwardly as this problem most often can be solved by separating the double integral into simple ones. It is no longer the case when both random variables are dependent. We address here this other case for the exponential bivariate.

Let us define a general form of objective function with two decision variables $x$ and $y$ and two random variables $Q$ and $P$ which can range over a probability plane $\Omega=\Omega_{q} \times \Omega_{p}$ with $x$ of the same nature as $q$ an outcome of $\Omega_{q}$ and y of the same nature as $p$ an outcome of $\Omega_{p}$ :

$$
C_{\Omega}(x, y \mid q, p)=\alpha q+\beta p+\gamma q p+\delta q^{2}+\varepsilon
$$

for a given set of $\alpha, \beta, \gamma, \delta, \varepsilon$ function of the decision variables $x$ and $y$ in any form. We consider the behavior of this objective function over a bidimensional probability plane $\Omega$ subdivided into regions with varying bounds, (see figure 1 ). We are interested in particular in $\Omega^{2}=[0 ; x] \times[0 ; y]$, where both intervals can be finite or infinite. $A$ represents the resulting outcome of the objective function in the probability plane. We have to give an analytical expression for $A$, the expected value of the function to be optimized. In classical form, the expression $A$ is written:

$$
A=E\left[C_{\Omega}(x, y \mid Q, P)\right]=\iint_{\Omega} C_{\Omega}(x, y) f\left(q, p, \lambda_{1}, \lambda_{2}, \rho\right) d q d p .
$$




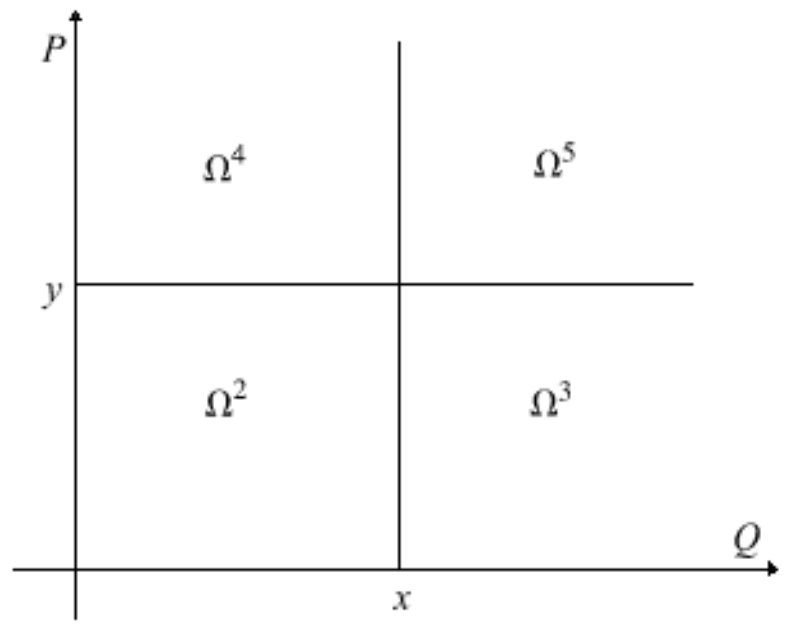

Fig. 1. The domains $\Omega^{k}, k=2,3,4,5 ; \Omega^{1}$ is the complete quarter plane.

Our concern is the evaluation of $A$ for any tuple $\{x, y\} \in \mathbb{R}^{2 *}$. We summarize a few properties of double integrals of the modified Bessel function in annex A.

\subsection{Expressing the cost function in a specific example for $\boldsymbol{C}_{\boldsymbol{\Omega}}$}

For the evaluation of the expression $A$ defined in (2.5) using the objective function $C_{\Omega}(x, y \mid q, p)$ of (2.4), we need the expressions

$$
A_{i j}=\iint_{\Omega} q^{i} p^{j} f\left(q, p, \lambda_{1}, \lambda_{2}, \rho\right) d q d p
$$

for the pairs

$$
(i, j)=(0,0),(1,0),(0,1),(1,1),(2,0) .
$$

\section{Evaluation of $A_{i j}$ in terms of the $K$-function and its derivatives}

We evaluate the expression $A_{i j}$ of (2.6) and use the notation

$$
\lambda=\frac{\lambda_{1}}{1-\rho}, \quad \mu=\frac{\lambda_{2}}{1-\rho} .
$$

To evaluate $A_{i j}$ it is convenient to consider for each function 5 domains, namely the regions $\Omega_{i}$ for $i \in 1, \ldots, 4$ represented in 1 . The following integrals need to be evaluated

$$
A_{i j}^{k}=\iint_{\Omega^{k}} q^{i} p^{j} f(q, p) d q d p
$$

where

$$
f(q, p)=(1-\rho) \lambda \mu e^{-\lambda q-\mu p} I_{0}(2 \sqrt{\rho \lambda \mu p q}) .
$$


However, we take $\lambda=\mu=1$, because general values of these quantities follow from rescaling $q$ and $p$. That is, we take

$$
f(q, p)=(1-\rho) e^{-q-p} I_{0}(2 \sqrt{\rho p q}) .
$$

It is possible to express the integrals over one of the domains $\Omega^{k}$ in terms of the integrals over other domains. For example, the integrals over $\Omega^{5}$ follow from those over $\Omega^{k}, k=1,2,3,4$. However, when $x$ and $y$ are large, the integrals over $\Omega^{5}$ may become exponentially small. Consequently, for numerical computations we need all integrals over all $\Omega$ - domains. In the appendix A, we have given some demonstrations of various integrals and functions which will play a role in the following. Among them, the following integrals will be used frequently

$$
\begin{aligned}
& \int_{0}^{\infty} e^{-\alpha t} I_{0}(2 \sqrt{\beta t}) d t=\frac{1}{\alpha} e^{\beta / \alpha} \\
& \int_{0}^{\infty} t e^{-\alpha t} I_{0}(2 \sqrt{\beta t}) d t=\frac{\alpha+\beta}{\alpha^{3}} e^{\beta / \alpha} \\
& \int_{0}^{\infty} t^{2} e^{-\alpha t} I_{0}(2 \sqrt{\beta t}) d t=\frac{2 \alpha^{2}+4 \alpha \beta+\beta^{2}}{\alpha^{5}} e^{\beta / \alpha},
\end{aligned}
$$

where the first one follows from (A.4), the second and third ones from differentiation of the first one with respect to $\alpha$. It is also useful to have a different representation of the $L$-function. By using the expansion (1.2) of the Bessel function in (A.1), we can express it in a different way from Temme (1996) as:

$$
L(x, y, p)=(1-p) \sum_{k=0}^{\infty} p^{k} \frac{\gamma(k+1, x)}{k !} \frac{\gamma(k+1, y)}{k !}
$$

where $\gamma(a, z)$ is the incomplete gamma function (see (Temme, 1996, Chapter 11))

$$
\gamma(a, z)=\int_{0}^{z} t^{a-1} e^{-t} d t, \quad \Re a>0,
$$

where $\Re a$ is the real part of $a$. In a similar way

$$
K(x, y)=e^{-y} \sum_{k=0}^{\infty} \frac{\gamma(k+1, x)}{k !} \frac{y^{k}}{k !}
$$

We will show that the functions $A_{i j}^{k}, i+j>0$ can be obtained by using derivatives of the $L$-function, and that, because of (A.2), all $A_{i j}^{k}$ can be obtained from derivatives of the $K$-function. Useful relations are

$$
\frac{\partial K(x, y)}{\partial x}=e^{-x-y} I_{0}(2 \sqrt{x y}), \quad \frac{\partial K(x, y)}{\partial y}=-\sqrt{x / y} e^{-x-y} I_{1}(2 \sqrt{x y}),
$$


The first relation follows from (A.3) and the second one from (A.7), which gives

$$
\frac{\partial K(x, y)}{\partial y}=-e^{-x-y} \frac{\partial I_{0}(2 \sqrt{x y})}{\partial y} .
$$

Using $I_{0}^{\prime}(z)=I_{1}(z)$ (see 9.6.27 of Abramovitz \& Stegun (1964)), we obtain the second relation in (3.9). Using (A.7) we write the $L$-function of (A.2) in a more symmetric form as

$$
L(x, y, \rho)=1-e^{-x-y} I_{0}(2 \sqrt{\rho x y})-e^{-x(1-\rho)} K(y, \rho x)-e^{-y(1-\rho)} K(x, \rho y) .
$$

\subsection{The integrals $\boldsymbol{A}_{\mathbf{0 0}}^{\boldsymbol{k}}$}

For $A_{00}^{1}$ we have using the first integral in (3.5)

$$
A_{00}^{1}=(1-\rho) \int_{0}^{\infty} e^{-p} \int_{0}^{\infty} e^{-q} I_{0}(2 \sqrt{\rho p q}) d q d p=(1-\rho) \int_{0}^{\infty} e^{-(1-\rho) p} d p=1 .
$$

For $A_{00}^{2}$ we have using (A.1)

$$
A_{00}^{2}=L(x, y, \rho)
$$

and the relation with the $K$-function follows from (A.2) or (3.11). Next, for $A_{00}^{3}$ we have

$$
\begin{aligned}
A_{00}^{3} & =\int_{0}^{y} \int_{x}^{\infty} f(q, p) d q d p \\
& =\int_{0}^{y} \int_{0}^{\infty} f(q, p) d q d p-\int_{0}^{y} \int_{0}^{x} f(q, p) d q d p \\
& =(1-\rho) \int_{0}^{y} e^{-(1-\rho) p} d p-A_{00}^{2} \\
& =1-e^{-(1-\rho) y}-A_{00}^{2} . \\
& =e^{-(1-\rho) x} K(y, \rho x)-e^{-(1-\rho) y} K(\rho y, x) .
\end{aligned}
$$

Similarly,

$$
\begin{aligned}
A_{00}^{4} & =\int_{y}^{\infty} \int_{0}^{x} f(q, p) d q d p \\
& =1-e^{-(1-\rho) x}-A_{00}^{2} \\
& =e^{-(1-\rho) y} K(x, \rho y)-e^{-(1-\rho) x} K(\rho x, y) .
\end{aligned}
$$


Finally,

$$
\begin{aligned}
A_{00}^{5} & =\int_{y}^{\infty} \int_{x}^{\infty} f(q, p) d q d p \\
& =1-A_{00}^{2}-A_{00}^{3}-A_{00}^{4} \\
& =e^{-(1-\rho) y} K(\rho y, x)+e^{-(1-\rho) x} K(y, \rho x)+e^{-x-y} I_{0}(2 \sqrt{\rho x y}) .
\end{aligned}
$$

\subsection{The integrals $\boldsymbol{A}_{\mathbf{1 0}}^{\boldsymbol{k}}$}

For $A_{10}^{1}$ we have, using the second integral in (3.5),

$$
\begin{aligned}
A_{10}^{1} & =(1-\rho) \int_{0}^{\infty} e^{-p} \int_{0}^{\infty} q e^{-q} I_{0}(2 \sqrt{\rho p q}) d q d p \\
& =(1-\rho) \int_{0}^{\infty} e^{-(1-\rho) p}(1+\rho p) d p=\frac{1}{1-\rho}
\end{aligned}
$$

For $A_{10}^{2}$ we have

$$
\begin{aligned}
A_{10}^{2} & =\int_{0}^{y} \int_{0}^{x} q f(q, p) d q d p \\
& =(1-\rho) \sum_{k=0}^{\infty} \frac{\rho^{k}}{k ! k !} \int_{0}^{x} u^{k+1} e^{-u} d u \int_{0}^{y} v^{k} e^{-v} d v
\end{aligned}
$$

giving

$$
A_{10}^{2}=(1-\rho) \sum_{k=0}^{\infty} \rho^{k} \frac{\gamma(k+2, x)}{k !} \frac{\gamma(k+1, y)}{k !} .
$$

Using the recursion

$$
\gamma(a+1, z)=a \gamma(a, z)-z^{a} e^{-z}
$$

we obtain

$$
\begin{aligned}
A_{10}^{2}= & (1-\rho) \sum_{k=0}^{\infty}(k+1) \rho^{k} \frac{\gamma(k+1, x)}{k !} \frac{\gamma(k+1, y)}{k !}- \\
& (1-\rho) x e^{-x} \sum_{k=0}^{\infty} \frac{\rho^{k} x^{k}}{k !} \frac{\gamma(k+1, y)}{k !} .
\end{aligned}
$$

To evaluate the first series we use (3.6) and for the second one we use (3.8). This gives

$$
A_{10}^{2}=(1-\rho) \frac{\partial}{\partial \rho}\left[\frac{\rho L(x, y, \rho)}{1-\rho}\right]-(1-\rho) x e^{-x(1-\rho)} K(y, \rho x) .
$$


Using the derivatives (3.9) and the relations (A.6),(A.7), we obtain

$$
\begin{aligned}
(1-\rho) A_{10}^{2}= & 1-e^{-x-y} I_{0}(\xi)+\frac{1}{2}(1-\rho) \xi e^{-x-y} I_{1}(\xi) \\
& -(1+w) e^{-w} K(y, \rho x)-(1+\rho z) e^{-z} K(x, \rho y),
\end{aligned}
$$

where

$$
\xi=2 \sqrt{\rho x y}, \quad w=(1-\rho) x, \quad z=(1-\rho) y .
$$

Next, for $A_{10}^{3}$ we have

$$
\begin{aligned}
A_{10}^{3}= & \int_{0}^{y} \int_{x}^{\infty} q f(q, p) d q d p \\
= & \int_{0}^{y} \int_{0}^{\infty} q f(q, p) d q d p-\int_{0}^{y} \int_{0}^{x} q f(q, p) d q d p \\
= & (1-\rho) \int_{0}^{y} e^{-(1-\rho) y}(1+\rho p) d p-A_{10}^{2} \\
= & \gamma(1,(1-\rho) y)-\frac{\rho}{1-\rho} \gamma(2,(1-\rho) y)-A_{10}^{2} \\
= & \frac{1}{1-\rho}\left[1-(1+\rho(1-\rho) y) e^{-(1-\rho) y}-A_{10}^{2}\right] \\
= & \frac{1}{1-\rho}\left\{(1+w) e^{-w} K(y, \rho x)-(1+\rho z) e^{-z} K(\rho y, x)\right. \\
& \left.\quad-e^{-x-y}\left[\rho z I_{0}(\xi)+\frac{1}{2}(1-\rho) \xi I_{1}(\xi)\right]\right\} .
\end{aligned}
$$

Similarly,

$$
\begin{aligned}
& A_{10}^{4}= \int_{y}^{\infty} \int_{0}^{x} q f(q, p) d q d p \\
&=\frac{1}{1-\rho}\left\{(1+\rho z) e^{-z} K(x, \rho y)-(1+w) e^{-w} K(\rho x, y)\right. \\
&\left.\quad-e^{-x-y}\left[w I_{0}(\xi)+\frac{1}{2}(1-\rho) \xi I_{1}(\xi)\right]\right\}
\end{aligned}
$$

Finally,

$$
\begin{aligned}
& A_{10}^{5}= \int_{y}^{\infty} \int_{x}^{\infty} q f(q, p) d q d p \\
&=\frac{1}{1-\rho}\left\{(1+\rho z) e^{-z} K(\rho y, x)+(1+w) e^{-w} K(\rho x, y)\right. \\
&\left.\quad+e^{-x-y}\left[(1+w+\rho z) I_{0}(\xi)+\frac{1}{2}(1-\rho) \xi I_{1}(\xi)\right]\right\},
\end{aligned}
$$




\subsection{The integrals $\boldsymbol{A}_{\mathbf{0 1}}^{\boldsymbol{k}}$}

These results follow from $\S 3.2$ by interchanging $x$ and $y$ (and $w$ and $z$ ) throughout. We give these result without further details. For $A_{01}^{1}$ we have

$$
A_{01}^{1}=(1-\rho) \int_{0}^{\infty} p e^{-p} \int_{0}^{\infty} e^{-q} I_{0}(2 \sqrt{\rho p q}) d q d p=\frac{1}{1-\rho}
$$

Next, for $A_{01}^{2}$ :

$$
\begin{aligned}
A_{01}^{2} & =\int_{0}^{y} \int_{0}^{x} p f(q, p) d q d p \\
& =(1-\rho) \frac{\partial}{\partial \rho}\left[\frac{\rho L(x, y, \rho)}{1-\rho}\right]-(1-\rho) y e^{-y(1-\rho)} K(x, \rho y) .
\end{aligned}
$$

In terms of the $K$-function this becomes (cf. (3.23))

$$
\begin{aligned}
(1-\rho) A_{01}^{2}= & 1-e^{-x-y} I_{0}(\xi)+\frac{1}{2}(1-\rho) \xi e^{-x-y} I_{1}(\xi) \\
& -(1+\rho w) e^{-w} K(y, \rho x)-(1+z) e^{-z} K(x, \rho y)
\end{aligned}
$$

For the other functions we have

$$
\begin{aligned}
A_{01}^{3}= & \int_{0}^{y} \int_{x}^{\infty} p f(q, p) d q d p \\
= & \frac{1}{1-\rho}\left\{(1+\rho w) e^{-w} K(y, \rho x)-(1+z) e^{-z} K(\rho y, x)\right. \\
& \left.\quad-e^{-x-y}\left[z I_{0}(\xi)+\frac{1}{2}(1-\rho) \xi I_{1}(\xi)\right]\right\}
\end{aligned}
$$




\subsection{The integrals $\boldsymbol{A}_{\mathbf{1 1}}^{\boldsymbol{k}}$}

For $A_{11}^{1}$ we have

$$
A_{11}^{1}=(1-\rho) \int_{0}^{\infty} p e^{-p} \int_{0}^{\infty} q e^{-q} I_{0}(2 \sqrt{\rho p q}) d q d p=\frac{1+\rho}{(1-\rho)^{2}} .
$$

For $A_{11}^{2}$ we have

$$
\begin{aligned}
A_{11}^{2} & =\int_{0}^{y} \int_{0}^{x} q p f(q, p) d q d p \\
& =(1-\rho) \sum_{k=0}^{\infty} \rho^{k} \frac{\gamma(k+2, x)}{k !} \frac{\gamma(k+2, y)}{k !} .
\end{aligned}
$$

By using (3.20) we write

$$
A_{11}^{2}=B_{1}+B_{2}+B_{3}+B_{4}
$$

where

$$
\begin{aligned}
& B_{1}=(1-\rho) \sum_{k=0}^{\infty}(k+1)^{2} \rho^{k} \frac{\gamma(k+1, x)}{k !} \frac{\gamma(k+1, y)}{k !}, \\
& B_{2}=-(1-\rho) y e^{-y} \sum_{k=0}^{\infty}(k+1) \frac{\rho^{k} y^{k}}{k !} \frac{\gamma(k+1, x)}{k !}, \\
& B_{3}=-(1-\rho) x e^{-x} \sum_{k=0}^{\infty}(k+1) \frac{\rho^{k} x^{k}}{k !} \frac{\gamma(k+1, y)}{k !}, \\
& B_{4}=(1-\rho) x y e^{-x-y} \sum_{k=0}^{\infty} \frac{\rho^{k} x^{k} y^{k}}{k ! k !} .
\end{aligned}
$$

For $B_{1}$ we obtain

$$
B_{1}=(1-\rho) \frac{\partial}{\partial \rho}\left[\rho \frac{\partial}{\partial \rho}\left\{\rho \frac{L(x, y, \rho)}{1-\rho}\right\}\right],
$$

which can be evaluated in the form

$$
\begin{aligned}
(1-\rho)^{2} B_{1}= & 1+\rho+c_{01} e^{-x-y} I_{0}(\xi)+c_{11} e^{-x-y} I_{1}(\xi) \\
& -\kappa_{11} e^{-x(1-\rho)} K(y, \rho x)-\kappa_{21} e^{-y(1-\rho)} K(x, \rho y),
\end{aligned}
$$

where

$$
\begin{aligned}
& c_{01}=x y \rho(1-\rho)^{2}-1-\rho, \\
& c_{11}=\frac{1}{2} \xi(1-\rho)[\rho(1-\rho)(x+y)+2], \\
& \kappa_{11}=1+\rho+\rho x(1-\rho)(3-\rho)+\rho^{2} x^{2}(1-\rho)^{2}, \\
& \kappa_{21}=1+\rho+\rho y(1-\rho)(3-\rho)+\rho^{2} y^{2}(1-\rho)^{2} .
\end{aligned}
$$


The other $B_{j}$ are given by

$$
\begin{aligned}
B_{2} & =-(1-\rho) y e^{-y} \frac{\partial}{\partial \rho}\left[\rho e^{\rho y} K(x, \rho y)\right] \\
& =-(1-\rho) y\left[e^{-y(1-\rho)}(1+\rho y) K(x, \rho y)-\frac{1}{2} \xi e^{-x-y} I_{1}(\xi)\right], \\
B_{3} & =-(1-\rho) x e^{-x} \frac{\partial}{\partial \rho}\left[\rho e^{x} K(y, \rho x)\right] \\
& =-(1-\rho) x\left[e^{-x(1-\rho)}(1+\rho x) K(y, \rho x)-\frac{1}{2} \xi e^{-x-y} I_{1}(\xi)\right], \\
& B_{4}=(1-\rho) x y e^{-x-y} I_{0}(2 \sqrt{\rho x y}) .
\end{aligned}
$$

Collecting the results for $A_{11}^{2}$, we have

$$
\begin{aligned}
A_{11}^{2}= & \int_{0}^{y} \int_{0}^{x} q p f(q, p) d q d p \\
= & \frac{1}{(1-\rho)^{2}}\left\{1+\rho+e^{-x-y}\left[c_{0} I_{0}(\xi)+c_{1} I_{1}(\xi)\right]\right. \\
& \left.\quad-\kappa_{1} e^{-w} K(y, \rho x)-\kappa_{2} e^{-z} K(x, \rho y)\right\},
\end{aligned}
$$

where

$$
\begin{aligned}
& c_{0}=x y(1-\rho)^{2}-1-\rho, \\
& c_{1}=\frac{1}{2} \xi(1-\rho)[2+(1-\rho)(x+y)], \\
& \kappa_{1}=1+\rho+x(1-\rho)[1+\rho+x \rho(1-\rho)], \\
& \kappa_{2}=1+\rho+y(1-\rho)[1+\rho+y \rho(1-\rho)] .
\end{aligned}
$$

For the other functions we have (we use also (A.7))

$$
\begin{gathered}
A_{11}^{3}=\int_{0}^{y} \int_{x}^{\infty} q p f(q, p) d q d p=\int_{0}^{y} \int_{0}^{\infty} q p f(q, p) d q d p-A_{11}^{2}= \\
=\frac{1}{(1-\rho)^{2}}\left\{-e^{-x-y}\left[\left(c_{0}+\kappa_{2}\right) I_{0}(\xi)+c_{1} I_{1}(\xi)\right]\right. \\
\left.\quad+\kappa_{1} e^{-w} K(y, \rho x)-\kappa_{2} e^{-z} K(\rho y, x)\right\}, \\
A_{11}^{4}=\int_{y}^{\infty} \int_{0}^{x} q p f(q, p) d q d p \\
=\frac{1}{(1-\rho)^{2}}\left\{-e^{-x-y}\left[\left(c_{0}+\kappa_{1}\right) I_{0}(\xi)+c_{1} I_{1}(\xi)\right]\right. \\
\left.\quad-\kappa_{1} e^{-w} K(\rho x, y)+\kappa_{2} e^{-z} K(x, \rho y)\right\},
\end{gathered}
$$




$$
\begin{aligned}
A_{11}^{5}= & \int_{y}^{\infty} \int_{x}^{\infty} q p f(q, p) d q d p \\
= & \frac{1}{(1-\rho)^{2}}\left\{-3 e^{-x-y}\left[c_{0} I_{0}(\xi)+c_{1} I_{1}(\xi)\right]-\kappa_{1} e^{-w}-\kappa_{2} e^{-z}\right. \\
& \left.\quad+3 \kappa_{1} e^{-w} K(y, \rho x)+3 \kappa_{2} e^{-z} K(x, \rho y)\right\},
\end{aligned}
$$

where $\xi, w$ and $z$ are given in (3.24), and $c_{0}, c_{1}, \kappa_{1}, \kappa_{2}$ in (3.45).

\subsection{The integrals $\boldsymbol{A}_{\mathbf{2 0}}^{\boldsymbol{k}}$}

For $A_{20}^{1}$ we have using the third integral in (3.5)

$$
\begin{aligned}
A_{20}^{1} & =(1-\rho) \int_{0}^{\infty} e^{-p} \int_{0}^{\infty} q^{2} e^{-q} I_{0}(2 \sqrt{\rho p q}) d q d p \\
& =(1-\rho) \int_{0}^{\infty} e^{-(1-\rho) p}\left(2+4 \rho p+\rho^{2} p^{2}\right) d p=\frac{2}{(1-\rho)^{2}} .
\end{aligned}
$$

For the expression $A_{20}^{2}$, we find using (3.19) and (3.20)

$$
\begin{aligned}
A_{20}^{2} & =\int_{0}^{y} \int_{0}^{x} q^{2} f(q, p) d q d p \\
& =\frac{1-\rho}{\rho} \frac{\partial}{\partial \rho}\left[\frac{\rho^{2}}{1-\rho} A_{10}^{2}\right]-(1-\rho) x^{2} e^{-x(1-\rho)} K(y, \rho x),
\end{aligned}
$$

which can be evaluated in the form

$$
\begin{aligned}
(1-\rho)^{2} A_{20}^{2}= & 2+c_{0} e^{-x-y} I_{0}(\xi)+c_{1} e^{-x-y} I_{1}(\xi)+ \\
& \kappa_{1} e^{-x(1-\rho)} K(x, \rho y)+\kappa_{2} e^{-y(1-\rho)} K(y, \rho x),
\end{aligned}
$$

where

$$
\begin{aligned}
& c_{0}=-2+\frac{1}{4} \xi^{2}(1-\rho)^{2}, \\
& c_{1}=-\frac{1}{2} \xi(1-\rho)[\rho(1+x)-y \rho(1-\rho)-3-x], \\
& \kappa_{1}=-2+\rho y(1-\rho)[2 \rho-4-\rho y(1-\rho)], \\
& \kappa_{2}=-2-x(1-\rho)[2+x(1-\rho)] .
\end{aligned}
$$

\section{Rewriting using objective functions}

- The case when the expected value is estimated over the entire $\Omega$ 
$A$ from (2.6) using (2.4) becomes:

$$
A=\iint_{\Omega}\left(\alpha x+\beta y+\gamma x y+\delta x^{2}+\varepsilon\right) f\left(x, y, \lambda_{1}, \lambda_{2}, \rho\right) d x d y
$$

From (2.6), we can write:

$$
A=\alpha A_{10}+\beta A_{01}+\gamma A_{20}+\varepsilon A_{00}
$$

and by using the values found in (3.12), (3.28), (3.17), (3.49), we get

$$
E\left[C_{\Omega}(x, y \mid Q, P)\right]=(\alpha+\beta)\left(\frac{1}{1-\rho}\right)-\gamma\left(\frac{1}{(\rho-1)^{3}}\right)+\varepsilon
$$

This last equation is only a function of the parameters $\alpha, \beta, \gamma, \varepsilon$ which are expressed only in terms of $x$ and $y$.

- The case when the decision variables are part of the definition of the probability plane

This is the case when the objective function's expected value has to be estimated with decision variables involved in the definition of the boundaries of the probability plane: $\Omega^{2}=[0, x] \times[0, y]$, the expectation of the objective function becomes:

$$
E\left[C_{\Omega}(x, y \mid Q, P)\right]=\alpha A_{10}^{2}+\beta A_{01}^{2}+\gamma A_{11}^{2}+\delta A_{20}^{2}+\varepsilon A_{00}^{2} .
$$

This solves the problem. We now have expressed the objective function in every region of the positive quarter plane as an analytical closed form expression without double integrals expressed only using the $K$ - function and the modified Bessel function of the first kind.

For numerical testing or numerical illustrations, we have developed some algorithms specifically for Brusset \& Temme (2005) to enable extensive numerical testing of values for these variables in substantially shorter computer time. These algorithms have been included in appendix as routines for both Mathematica 5.0 (B) as well as Maple (B.1) using approximations for the modified Bessel function of the first kind.

\section{Conclusion}

We have shown how to circumvent the difficulties of the double integral in the case of dependent exponential bivariates. With these expressions, instances of decision processes with polynomial objective functions of any degree involving 
two decision variables both in the function itself and in the borders of the applicable domain involving two dependant random variables can be expressed in analytical form. The optimization of the expected objective functions usually become very quickly intractable, however, this method should reduce the difficulties in several instances. Moreover, the routines in Mathematica and Maple help to establish numerical results in manageable computer time.

These methods can be applied to the problems such as the case of a manufacturer and a retailer in the supply chain management efficiency mechanisms with two exponentially distributed variables. We feel that other Operational Research problems which have not occurred to us might also benefit with this approach. The routines spelt out in the annex allow to reduce substantially the calculation time to solve numerical applications. 


\section{References}

\section{References}

Abramovitz, M., \& Stegun, I.A. 1964. Handbook of mathematical functions with formulas, grpahs and mathematical tables. 55. Washington, DC: U.S. Government Printing Office. Paperback edition published by Dover, New York.

Brusset, Xavier, \& Temme, Nico M. 2005. Impact of information and coordination on transport procurement. In: De Koster, Rene (ed), Proceedings of the CEMS European Supply Chain Seminar 2004. Copenhagen, Denmark: Copenhagen Business School Press, for Community of European Management Schools.

Downton, F. 1970. Bivariate exponential distributions in reliability theory. Journal of Royal Statistical Society, B 32, 408-417.

Iliopoulos, George. 2003. Estimation of parametric functions in Downton's bivariate exponential distribution. Journal of statistical planning and inference, 117, 169-184.

KibBle, W.F. 1941. A two-variate gamma type distribution. Sankhya, 5, $137-150$.

Kleindorfer, Paul R., \& Wu, D.J. 2003. Integrating long- and shortterm contracting via business-to-business exchanges for capital-intensive industries. Management Science, 49(11), 1597-1615.

Kotz, S., Balakrishnan, N., \& Johnson, N.L. 2000. Continuous multivariate distributions. 2nd edn. Vol. 1. New York, NY: John Wiley \& Sons.

Lariviere, Martin. 1999. Supply Chain contracting and stochastic demand. Chap. 8, pages 234-267 of: TAyur, Sridhar, Ganeshan, Ram, \& MaGAZINe, Michael (eds), Quantitative models for Supply Chain Management. Dordrecht, Holland: Kluwer Academic.

LASSEY, K.R. 1982. On the computation of certain integrals containing the modified Bessel function $I_{0}(\xi)$. Mathematics of Computation, 39(160), 625637 .

Luke, Yudell LeO. 1962. Integrals of Bessel functions. New York, NY: McGraw-Hill.

Marshal, Albert, \& Olkin, Ingram. 1967. A multivariate exponential distribution. American Statistical Association Journal, March, 30-44.

Moran, P.A.P. 1967. Testing for correlation between non-negatie variates. Biometrika, 54, 385-394.

Seifert, Ralf, Thonemann, Ulrich, \& Hausman, Warren. 2004. Op- 
timal Procurement strategies for online spot markets. European Journal of Operational Research, 152(3), 781-799.

Spinler, Stefan, Huchzermeier, Arnd, \& Kleindorfer, PAUL R. 2003 (January). The valuation of options on capacity in the presence of state-contingent demand. EURO Management, http://opim.wharton.upenn.edu/home/pdf/seminars/previous/ spinler valuation_options.pdf.

Temme, Nico. 1986. A double integral containing the modified Bessel function: asymptotics and computation. Mathematics of Computation, 47(176), 683-691.

Temme, Nico. 1996. Special functions: an introduction the classical functions of mathematical physics. New York, NY: John Wiley \& Sons.

Wu, D.J., Kleindorfer, Paul R., \& Zhang, J.E. 2001a. Optimal bidding and contracting strategies in the deregulated electric power marketplace: Part II. In: JR, R.H. Sprague (ed), Proceedings of the 34 th Annual HAwaii International Conference on Systems Sciences. Los Alamitos, California, USA: IEEE Computer Society Press.

Wu, D.J., Kleindorfer, Paul R., \& Sun, Y. 2001b (June). Optimal capacity expansion in the presence of capacity options. Working Paper, Department of Operations and Information Management, the Wharton School, University of Pennsylvania, http://opim.wharton.upenn.edu/risk/downloads/01-30-PK.pdf.

Wu, D.J., Kleindorfer, Paul R., \& Zhang, Jin E. 2002. Optimal bidding and contracting strategies for capital-intensive goods. European Journal of Operational Research, 657-676. 


\section{A Integrals of Bessel functions}

The following integral, first discussed in Lassey (1982), has been studied at length in Temme (1986)

$$
L(x, y, p)=(1-p) \int_{0}^{x} \int_{0}^{y} e^{-u-v} I_{0}(2 \sqrt{p u v}) d u d v, \quad p \geq 0
$$

and plays a crucial role in the following analysis. For several properties we refer to Temme (1986); see also Luke (1962). This function can be written as

$$
L(x, y, p)=\left(1-e^{p y-y}\right)+e^{p y-y} K(p y, x)-e^{p x-x} K(y, p x)
$$

where

$$
K(x, y)=e^{-y} \int_{0}^{x} e^{-u} I_{0}(2 \sqrt{u y}) d u=1-e^{-y} \int_{x}^{\infty} e^{-u} I_{0}(2 \sqrt{u y}) d u .
$$

This complementary property follows from

$$
e^{y}=\int_{0}^{\infty} e^{-u} I_{0}(2 \sqrt{y u}) d u
$$

(see 29.3.81 of Abramovitz \& Stegun (1964)). The starting point in Temme (1986) is the function

$$
I(x, y)=\int_{0}^{x} \int_{0}^{y} e^{-u-v} I_{0}(2 \sqrt{u v}) d u d v .
$$

We have $I(x, y)=-\partial L(x, y, p) / \partial p$ at $p=1$ and

$$
I(x, y)=x+(y-x) K(x, y)-e^{-x-y}\left[\frac{1}{2} \xi I_{1}(\xi)+x I_{0}(\xi)\right], \quad \xi=2 \sqrt{x y} .
$$

Observe that $I(x, y)=I(y, x)$, and from this symmetry relation it follows that

$$
K(x, y)+K(y, x)=1-e^{-x-y} I_{0}(2 \sqrt{x y}),
$$

which is not given in Temme (1986). In (A.6), $I_{1}(\xi)$ is the modified Bessel function of the first kind of order one. The analysis in Temme (1986) is focused on the asymptotic properties of $I(x, y)$ for large values of $x$ and $y$. In fact the asymptotic properties of the function

$$
F(x, y)=K(x, y)+\frac{1}{2} e^{-x-y} I_{0}(2 \sqrt{x y})
$$

are studied. The symmetry rule for this function reads

$$
F(x, y)+F(y, x)=1 .
$$


The first term approximation for $F(x, y)$ given in Temme (1986) reads

$$
F(x, y) \sim \frac{\sqrt{x}+\sqrt{y}}{4 x y^{1 / 4}} \operatorname{erfc}(\sqrt{y}-\sqrt{x}), \quad y \geq x,
$$

where erfc $z$ denotes the complementary error function

$$
\operatorname{erfc} z=\frac{2}{\sqrt{\pi}} \int_{z}^{\infty} e^{-t^{2}} d t
$$

The above results are exposed here not only so that the reader does not have to refer in depth to the works cited (even though they are also apparent in Temme (1986)), but because they apply in the routines for the algorithms in the case of numerical calculations given in the appendix.

The approximation in (A.10) holds for large $y$, uniformly with respect to $x$, $0<\delta \leq x \leq y$, where $\delta$ is a fixed positive number. It gives the exact value $\frac{1}{2}$ when $x=y$, and for $y \gg x$ it is exponentially accurate. This follows from the estimate

$$
\operatorname{erfc} z \sim \frac{1}{z \sqrt{\pi}} e^{-z^{2}}, \quad z \rightarrow \infty .
$$

When $x \geq y$ we can use (A.9), and obtain

$$
F(x, y) \sim 1-\frac{\sqrt{x}+\sqrt{y}}{4 \sqrt{\sqrt{x y}}} \operatorname{erfc}(\sqrt{x}-\sqrt{y}), \quad x \geq y \geq \delta>0 .
$$

This non-uniform behaviour of $F(x, y)$ for large $x$ and $y$ also occurs for $K(x, y)$. In fact we have the following limiting values. First observe that from (A.7) it follows that

$$
K(x, x)=\frac{1}{2}\left[1-e^{-2 x} I_{0}(2 x)\right] .
$$

For large values of $x$ the right-hand side approaches $\frac{1}{2}$, because

$$
I_{0}(z) \sim \frac{e^{z}}{\sqrt{2 \pi z}}, \quad z \rightarrow \infty
$$

(see 9.7.1 of Abramovitz \& Stegun (1964) or (9.54) of Temme (1996)). For fixed $x$ and large $y$ we can use (A.3) and (A.15) to show that $K(x, y)$ tends to zero. From (A.3) and (A.4) we conclude that for fixed $y$ and large $x, K(x, y)$ tends to unity. In other words,

$$
\begin{aligned}
& \lim _{y \rightarrow \infty} K(x, y)=0, \quad x \text { fixed, } \\
& \lim _{x \rightarrow \infty} K(x, x)=\frac{1}{2}, \\
& \lim _{x \rightarrow \infty} K(x, y)=1, \quad y \text { fixed. }
\end{aligned}
$$




\section{B Mathematica routines}

To speed the calculations in Wolfram Research's Mathematica 5.0@), the following routines have been developed by the authors which still carry a precision of $10^{-8}$. The modified Bessel function of the first kind of order zero is calculated to the precision available in Mathematica for all numbers lesser than 50 and rounded by a ratio of polynomials for numbers exceeding 50 :

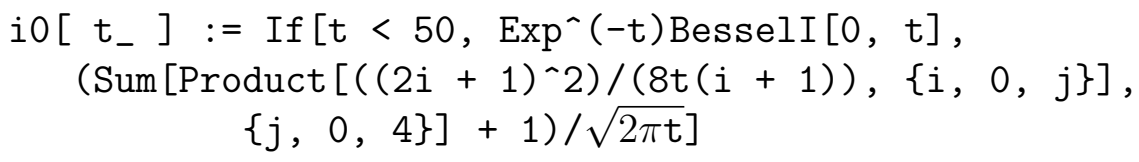

The modified Bessel function of order one of the first kind has the following routine:

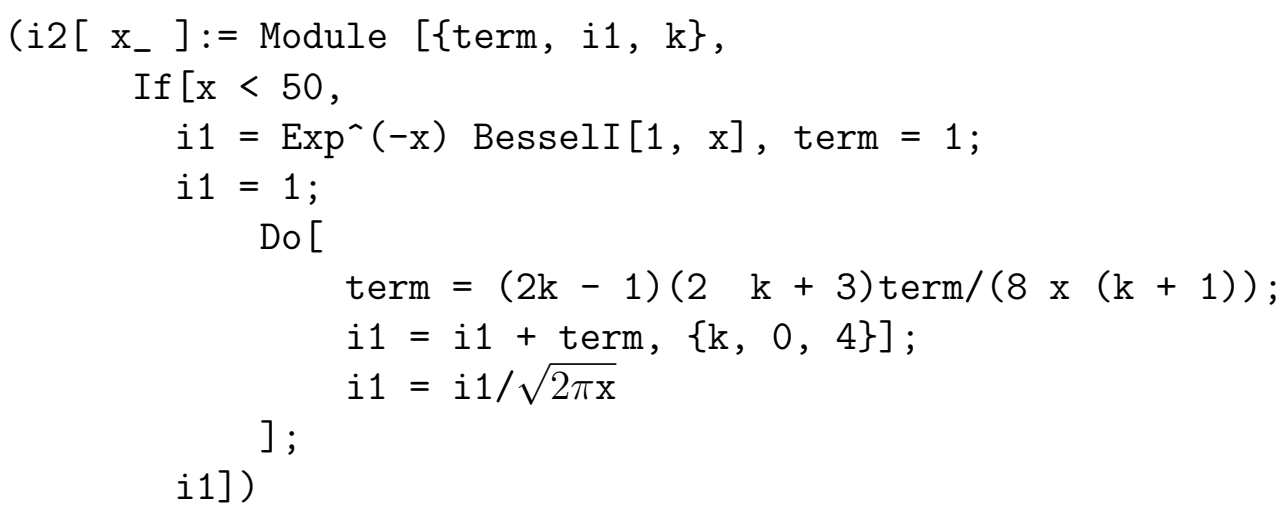

An algorithm for the $K$-function is:

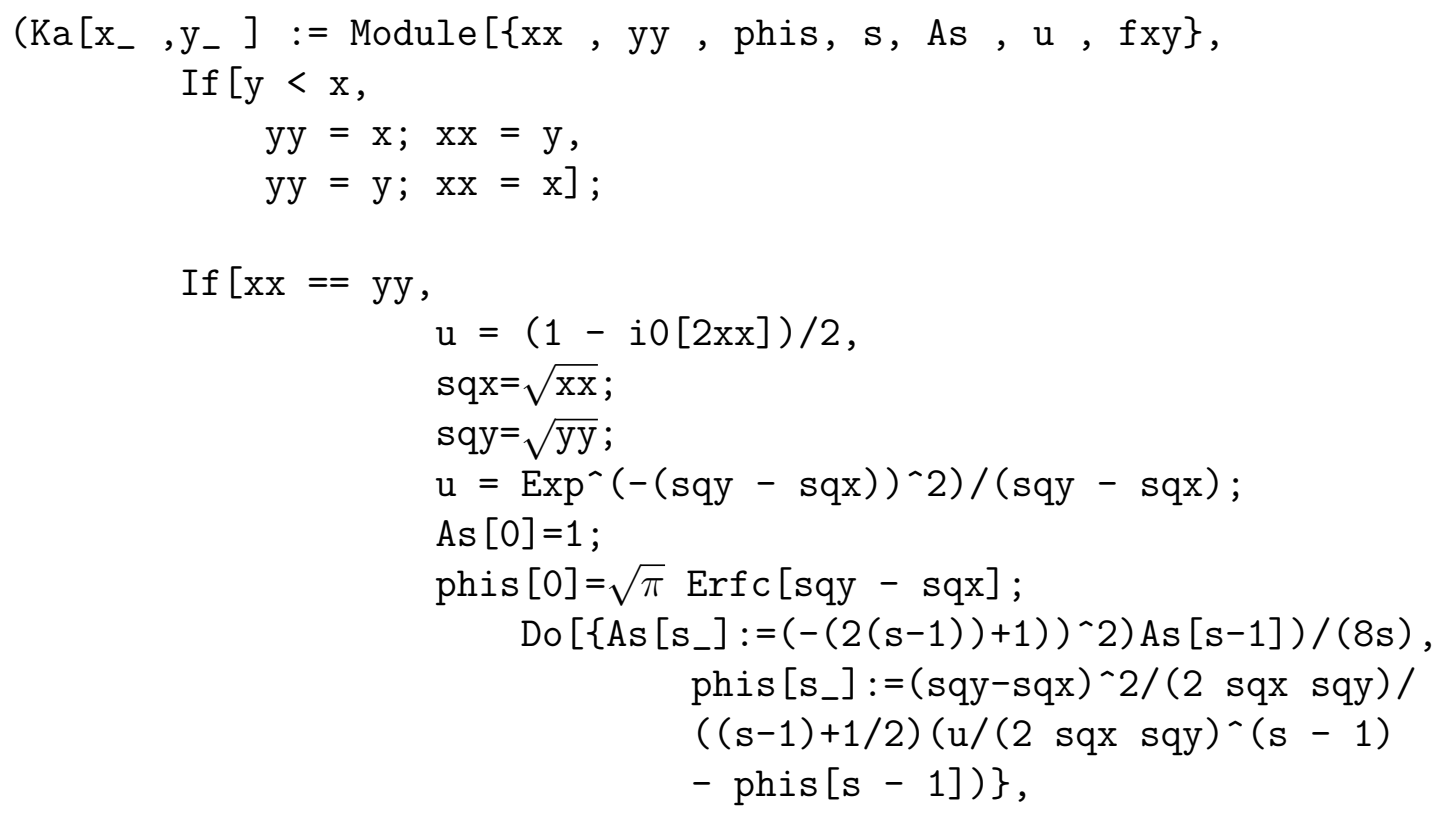




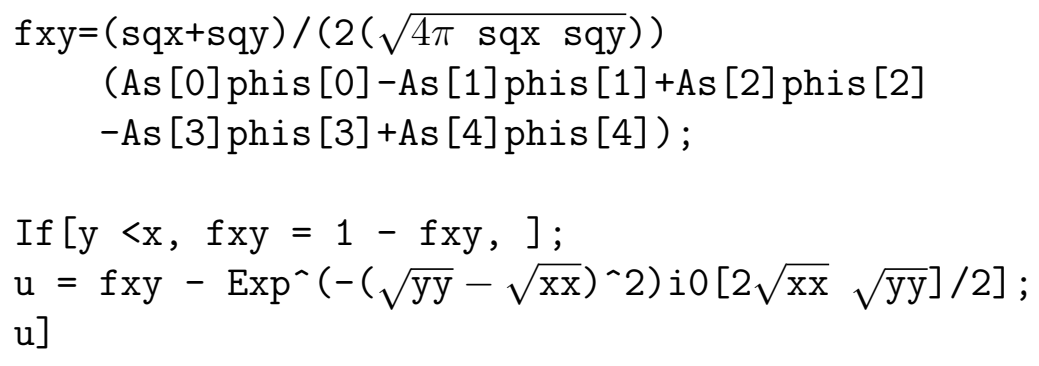

where Erfc is the complementary error function.

\section{B.1 Maple routines}

Below are the same routines in Maple programming language.

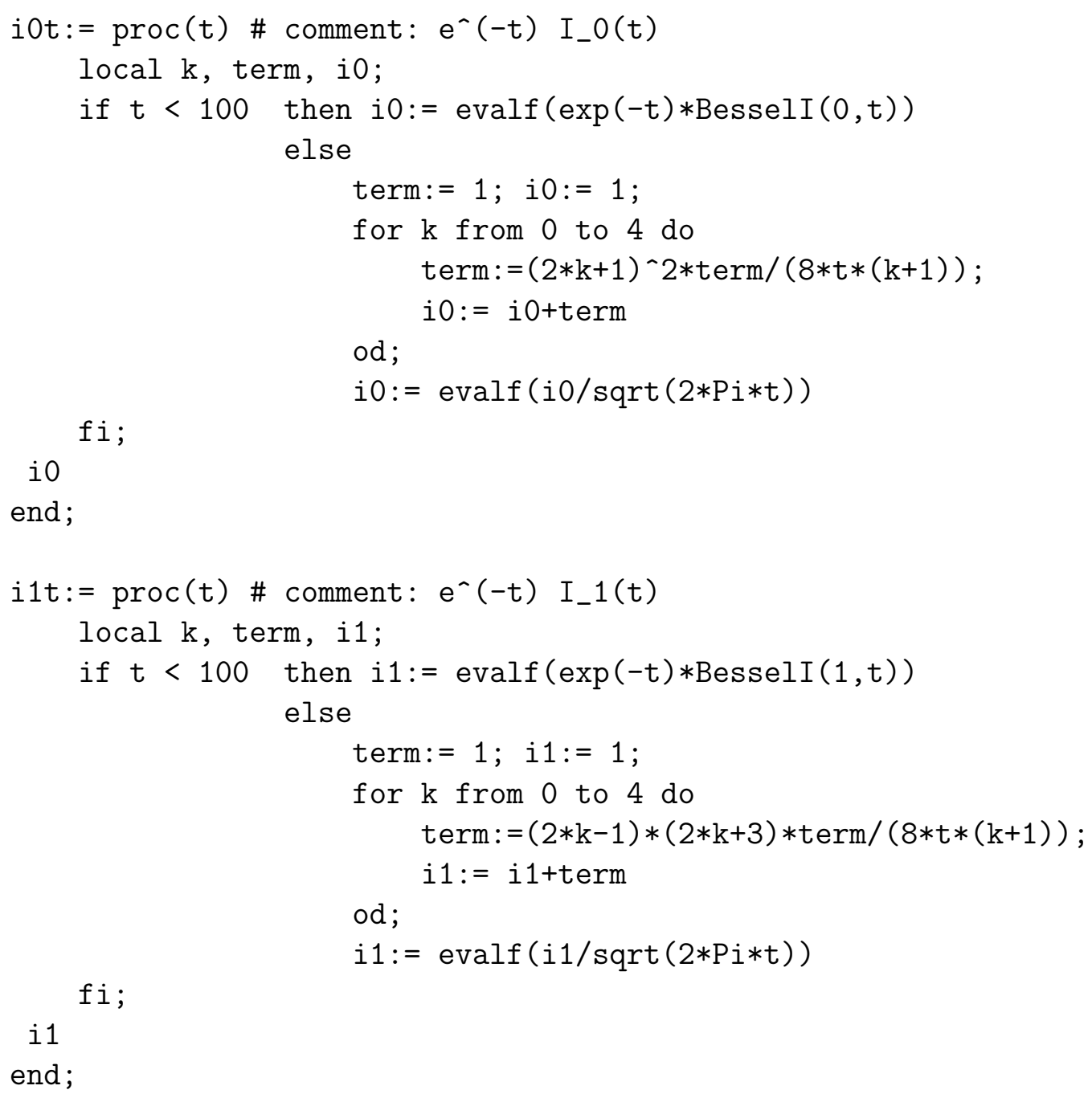




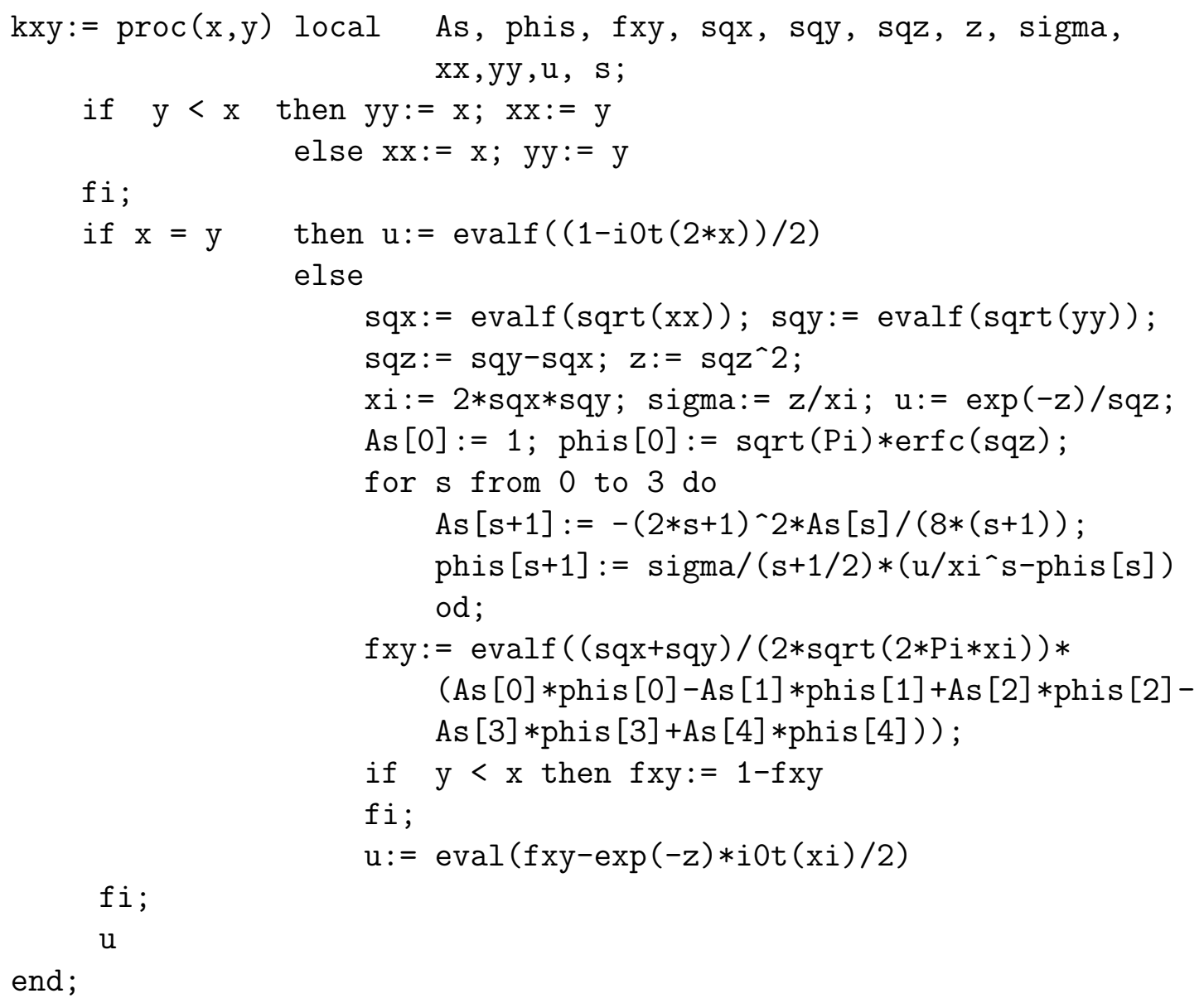

